\title{
A case of perioperative IABP use in a patient with AMI complicated with VSR.
}

\author{
Ervin Bejko *, Stavri Llazo, Esmerilda Bulku, Jonela Burimi, \\ Fation Hamiti, Alfred Ibrahimi, Saimir Kuci.
}

Received: 24 December 2021 / Accepted: 09 January 2022 / Published online: 20 January 2022

This article is published with open access at https://journal.astes.org.al

(C) The author(s) 2022. \& Copyright (C) 2022, the Albanian Society for Trauma and Emergency Surgery

(c) The Albanian Journal of Trauma and Emergency Surgery is an Open Access Journal. All articles are distributed under the terms of the Creative Commons Attribution Non-Commercial License: http://creativecommons.org/licenses/by-nc/4.0/) which permits unrestricted non-commercial use, distribution, and reproduction in any medium provided the original work is properly cited.

\begin{abstract}
Ventricular septal rupture (VSR) following myocardial infarction may lead to rapid clinical deterioration with pulmonary edema, hypotension, and a high early mortality. Inotropic agents can increase systemic output but at the possible cost of increased myocardial ischemia. In contrast, intra-aortic balloon pump (IABP) can decrease ischemia by raising coronary perfusion pressure and will reduce afterload. We are presenting the case of a 67-year-old male presented in the emergency department with strong chest pain, diagnosed as inferior acute myocardial infarction (AMI), in TTE visualised VSR with left-to-right shunt. After a few hours, the hemodynamic parameters deteriorated, with hemodynamic instability. The patient is placed on regime with IABP on 1: 1 ratio and on high-dose inotropic drug regime. Once hemodynamic stability is ensured, the patient prepares to perform the intervention. The intervention performed was closure of the VSR with patch Dacron via ventriculotomy of the inferior wall of the left ventricle. At all times, the patient remained associated with IABP, except for extracorporeal circulation time. After the intervention, the patient remained for several days bound to IABP, until hemodynamic stability was achieved, and the dose of inotropic drugs was reduced.
\end{abstract}

Keywords: VSR, AMI, IABP, Cardiac surgery.

\section{Abbreviations}

Ventricular Septal Rupture - VSR; , Intra-aortic Balloon Pump - IABP; Acute Myocardial Infarction - AMI; Percutaneous Coronary Intervention - PCI; Intensive Care Unite - ICU; Coronary Artery Disease - CAD; Transthoracic Echocardiography - TTE; Right Coronary Artery - RCA

Original article, no submission or publication in advance or in parallel

\footnotetext{
* Corresponding author: Ervin Bejko MD

$\bowtie$ bejko_ervin@yahoo.com

Cardiac Anaesthesia Department, University Hospital Centre "Mother Teresa" Tirana, ALBANIA
}

\section{Introduction}

Ventricular septal rupture is a rare but severe complication of acute myocardial infarction. The 30-day mortality rate of VSR is approximately $80 \%$. The incidence of VSR has substantially decreased since the widespread implementation of early reperfusion strategies, including primary percutaneous coronary intervention (PCI) and thrombolytic treatment, with a current estimate between $0.17 \%$ and $0.34 \%$. VSR occurs most often within the first $24 \mathrm{~h}$ after AMI, and the incidence of VSR is lower in patients who receive primary PCI compared with those who undergo delayed or elective PCI after a recent AMI.

Intra-aortic balloon pump is commonly used in patients with cardiogenic shock to temporarily stabilized hemodynamic disturbances. It can reduce the afterload of the left ventricle to decrease myocardial oxygen demand and increase the peak diastolic pressure. As a result, it can enhance coronary, cerebral, and renal perfusion, then subsequently raise cardiac output.

Here we report a case of VSR, and the importance of using IABP perioperatory for better hemodynamic performance and good results. 


\section{Case Report}

A 67-year-old man presented to emergency room with retrosternal constrictive chest pain, accompained by numbness of the left arm and profuse sweating. Patient refers that has 2 days with symptoms, but the day of presentation refers strong chest pain. He was admitted to emergency room, after that was transfered to Cardiac ICU service with diagnosis of inferior-AMI, in TTE was diagnosed with VSR, sinister-dexter shunt. Patient realised coronarography without heparine becouse of VSR, that result CAD 1-vessel, RCA cant be visualised good.

He was a nonsmoker, and has been treated for hypertension. Patient has family history for CAD. On physical examination, his blood pressure was 71/42 $\mathrm{mmHg}$ and his pulse rate was 66 beats/min. On auscultation systolic murmur was heard. The electrocardiogram showed normal sinus rhythm with ST-segment elevation in leads II, III and aVF, with Q-waves. A diagnosis of acute inferior myocardial infarction complicated with VSR was established.

After a few hours, the hemodynamic parameters deteriorated, with hemodynamic instability. The patient was transferred to Cardiac Surgery ICU service, connected with IABP on 1:1 ratio, and on high-dose of inotropic drugs, such as norepinephrine and dobutamine. Once hemodynamic stability was restored, patient was prepared for cardiac surgery. The intervention performed was closure of the VSR with patch Dacron via ventriculotomy of the inferior wall of the left ventricle. (fig. 1, 2)

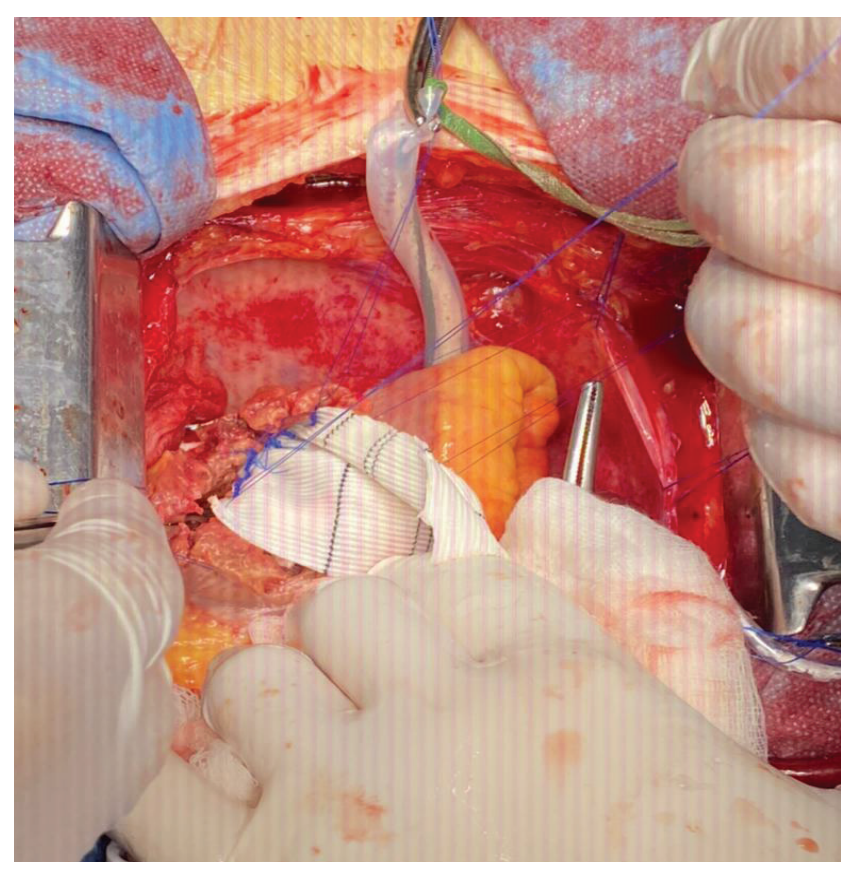

Figure 1- Inside view of VSR

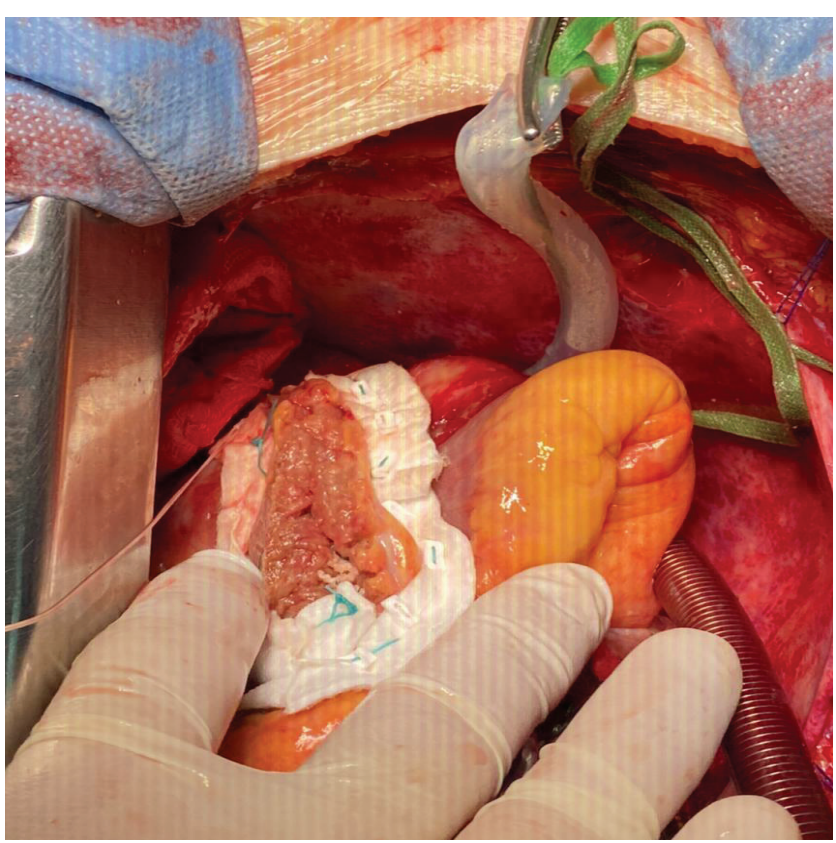

Figure 2 - Final result of Surgery

After the intervention, the patient was extubated the third day after surgery, remained for several days bound to IABP, until hemodynamic stability was achieved, arterial pressure, cardiac frecuency and diuresis was normal, and the dose of inotropic drugs was reduced day-by-day, until they stop one day before discharg from ICU.

\section{Dicussion}

Medical therapy for complications like VSR or mitral regurgitation secondary to papillary muscle dysfunction or rupture, often produces only a limited effect. In VSR, infusion of an inotropic agent may increase systemic output. However, this is associated with concomitant increase in left-toright shunting without change in the $\mathrm{P} / \mathrm{S}$ flow ratio, thereby resulting in a marked increase in left ventricular work. Agent which raise arterial pressure by increasing afterload augment pulmonary flow, increase left-to-right shunting, and may produce a critically low-output state.

Reduction in systemic resistance in ventricular septal rupture would favor systemic flow and decrease the left-toright shunt. However, in patients whose clinical condition is characterized by cardiogenic shock, any further reduction in systemic and coronary perfusion pressure is hazardous.

IABP can selectively reduce systemic resistance during left ventricular ejection thus producing the beneficial effect of a vasodilator without a reduction in mean arterial pressure. This is accompanied by an increase in coronary perfusion pressure which can reduced ischemic dysfunction. 


\section{Conclusion}

This case confirms the effect of IABP in a patient in whom mechanical lesions, such as VSR, have resulted in cardiogenic shock. IABP produced clinical and hemodynamic improvement. The peak improvement occurred within the first 24 hours of pumping. IABP does produce a sufficient level of hemodynamic stability to premit undergoing surgery. In this setting, the greatest chance for a therapeutic success lies in the early aplication of circulatory assistance.

In conclusion, we suggest that patients with postinfarction VSR with signs of cardiogenic shock should immediately receive IABP as a bridge to emergent surgical repair.

\section{Declarations}

Funding: Not applicable

Competing interests: None declared

Contributors: All authors designed the manuscript. SLl, $\mathrm{EB}, \mathrm{JB}, \mathrm{FH}, \mathrm{AI}$ and $\mathrm{SK}$ acquired the data, which all authors analysed. EB wrote the article, which all authors reviewed and approved for publication. All authors agreed to be accountable for all aspects of the work.

\section{References}

1- Transcatheter closure of ventricular septal rupture with prolonged support of intra-aortic balloon pump after primary PCI: a case report Chuan Yang, Yong Sun, Deling Zou, Zhaoqing Sun, Xinzhong Zhang, Guangsheng Su, Jing Qi \& Wenyue Pang.

2- Intraaortic Balloon Pumping for Ventricular Septal Defect or Mitral Regurgitation Complicating Acute Myocardial Infarction By HERMAN K. GOLD, M.D., ROBERT C. LEINBACH, M.D., CHARLES A. SANDERS, M.D., MORTIMER J. BUCKLEY, M.D., ELDRED D. MuNDTH, M.D., AND W. GERALD AuSTEN, M.D.

3- Intra-Aortic Balloon Pump Tahir M. Khan; Abdul H. Siddiqui.

4- Intraaortic Ballon Counterpulsation: Deciphering Its Effects on Coronary Flow*, DAVID O. WILLIAMS, MD, FACC.

5- Ventricular septal rupture complicating acute myocardial infarction: a contemporary review. Brandon M Jones, Samir R Kapadia, Nicholas G Smedira, Michael Robich, E Murat Tuz$\mathrm{cu}$, Venu Menon, Amar Krishnaswamy

6- Improving hemodynamics - Prolonged intra-aortic balloon pump usage in posterior ventricular septal rupture with right ventricle dysfunction. Karthik Raman, Thapo T Desugari, S Sowmya, Samuel S Angula, Kalaichelvan Uthayakumaran, Anbarasu Mohanraj Madras Medical Mission, Institute of Cardiovascular Disease, Chennai, Tamil Nadu, India 